ERYTHROCYTE GTP DEPLETION IN PNP DEFICIENCY

193 PRESENTING WITH HAEMOLYTIC ANAEMIA AND HYPOURICAEMIA

H.Anne Simmonds, Lynette D Fairbanks, Peter Timms. Guy's Hospital, London, UK, King Khalid Hospital,Jeddah, Saudi Arabia.

A 4-yr old Arab girl,referred for investigation of hypouricaemia and hypouricosuria discovered during studies for Coombes positive haemolytic anaemia,was found to excrete inosine, guanosine,deoxyinosine and deoxyguanosine in place of uric acid. The patient and a 10-month old brother were homozygous for purin nucleoside phosphorylase (PNP) deficiency. Three of the other four surviving siblings were heterozygotes, as were the parents who were first cousins. Another sibling had died of chicken-pox. The baby showed head-lag, the girl spastic diplegia.

As in our previous case, severe red cell GTP depletion associated with raised dGTP levels and grossly increased NaD levels, were noted. Red cell PP-ribose-P levels and lysate APRT activity were likewise increased. The clinical findings also accord with previous reports. In addition to immunodeficiency, anaemia and neurological complications (ataxia, spastic tetrasparesis) have been reported in 30-40z of cases.

The results suggest that patients with severe PNP deficiency may suffer the consequences of two enzyme defects - immunodeficiency due to an inability to metabolise toxic deoxynucleosides plus the neurological complications of hypoxanthine guanine phosphoribosyltransferase (HGPRT) deficiency because of a resultant lack of substrates for the latter. Red cell GTP depletion appears to be a consistent finding in inherited disorders associated with neurodevelopmental retardation.
MYOADENYLATE DEAMINASE DEFICIENCY AND MCARDLE'S DI-

196 SEASE: PLASMA ADENOSINE, INOSINE AND HYPOXANTHINE AFTER ISCHEMIC FOREARM EXERCISE.

Sietze P.T. Sinkeler, Ed M.G. Joosten, Ron A. Wevers, Rob A. Binkhorst and T. Lian 0ei.

Departments of Neurology, Physiology and Human Genetics, St. Rad boud Hospital, University of Nijmegen, Nijmegen, The Netheriands.

Plasma adenosine, inosine and hypoxanthine concentrations were assayed in 7 controls, 5 myoadenylate deaminase deficient (MADD)patients and 6 McArdle patients before and after ischemic forearm exercise. The MADD patients showed a significantly lower increase of plasma inosine and hypoxanthine following exercise as compared to the controls, indicating diminished adenine nucleotide catabolism in the exercising MADD muscle. In the McArdle patients the increase in plasma inosine and hypoxanthine after exercise did not differ significantly from the values measured in the controls. The plasma adenosine increase was very low in all test groups and there were no significant differences.
194 A CASE OF LESCH NYHAN SYNDROME WITH DELAYED ONSET OF SELF MUTILATION: A search for abnormal biochemical,immunological and cell growth characteristics in fibroblasts and neurotransmitters in urine.

Surjit Singh, Ingrid willers, Kurt Ulirich,H.Gustmann,

\section{A.Niederwieser and $\mathrm{H}$.Werner Goedde.}

Institute of Human Genetics, University of Hamburg (FRG) and Departments of Pediatrics, University of Münster, Kiel (FRG) and Zurich (Switzerland).

A 12 year patient with marked hyperuricemia, tetraspasticity, choreoatheotosis, dysarthia and mental retardation was recently referred for a sudden development of a rapidly increasing autoagressive behaviour. Erythrocyte HPRT activity was virtually nil. Lysates of fibroblasts showed values 2.68 of the controls.The hypoxanthine utilisation in intact fibroblasts was 38 in the patient and 358 in his mothers cells. Cell growth in the 8azaguanine and HAT was almost in the range of classical type and the mother showed heterozygosity. Surprisingly, the level of dopamine, $5 \mathrm{HT}$ and biopterin was significantly increased in the urine of unmedicated patient.Further biochemical data encompassing neurotransmitters, immunology, purine metabolism and cell selection would be presented.
SYNTHESIS AND TURNOVER OF PURTNE NUCLEOSIDE

197 PHOSPHORYLASE IN HUMAN LYMPHOCYTES Floyd F. Snyder, Kuldeep Neote and Eddie Kwan Departments of Pediatrics and Medical Biochemistry, Faculty of Medicine, University of Calgary, Calgary, Alberta, T2N 4N1, Canada

The synthesis of purine nucleoside phosphorylase (PNP) was examined during phytohemagglutinin stimulated T 1ymphocyte transformation using a polyclonal rabbit antibody to human erythrocyte PNP. Lymphocytes were pulsed at various times for 3 hours with $\left[{ }^{35} \mathrm{~S}\right]$ methionine and immunoprecipitated lysates were analyzed by SDS-polyacrylamide gel electrophoresis. The labelled 32,500 molecular weight species corresponding to PNP were cut out of the dried gels and counted. PNP synthesis increased greater than 10-fold during the first 12 hours of transformation and continued to a maximum of $30-$ fold. The rate of synthesis of PNP relative to total protein was $0.04 \%$ in unstimulated $\mathrm{T}$ 1ymphocytes and increased to $0.18 \% 12$ hours after stimulation and remained constant at $0.16-0.19 \%$ between 40 and 60 hours. These studies identify a $4-5$ fold preferential synthesis of PNP during the early stages of T 1ymphocyte transformation.

In other studies with the B lymphoblast WI-L2, proteins were labelled with $\left[{ }^{3} \mathrm{H}\right]$ leucine and during further culture in nonradioactive medium the half-lives for total protein and PNP were found to be 14.5 and 14.1 hours respectively.

Supported by the Medical Research Council of Canada Grant MT-6376.

ELEVATED ADENOSINE LEVELS IN NEWBORNS. Alf Sollevi, 198 ology and Pediatrics, Karolinska Hospital, Stockholm Sweden.

Hypoxia enhance tissue adenosine formation during experimental conditions. This study examines the levels of adenosine and its metabolites in umbilical plasma from newborns $(15-45$ sec after vaginal delivery), a clinical condition known to have low oxygen levels. The study was approved by the Ethics Commité.

Adenosine, inosine, hypoxanthine (by HPLC,1), blood gases and cathecholamines (NA and A, by HPLC,2) were determined in arterial and venous umbilical blood from 30 normal deliveries. $\mathrm{pH}$ was $7.25 \pm 0.06$, Bace excess $-5.7 \pm 2.8$ and arterial $\mathrm{PO}_{2} 2.0 \pm 0.6 \mathrm{kPa}$. Plasma NA (5.3-374 nM) and A (0.1-16.9 nM) showed marked elevation. Arterial plasma adenosine $0.61 \pm 0.09 \mu \mathrm{M}$ and inosine $0.67 \pm$ 0.07 MM was similar in venous plasma, corresponding to 4 times higher levels than. in adults $(1,3)$. Arterial hypoxanthine was $0.9 \mu M$ higher than the vein $(p<0.01)$.

This is the first clinical study demonstrating elevated adenosine and inosine levels during hypoxia and metabolic stress. It is proposed that adenosine may serve as a vascular and metabolic modulator in neonatals.

1) A. Sollevi et al. Acta Physiol Scand. 1984,121:165.

2) P. Hjemdahl et al. Life Sci 1979,25:131.

3) A. Sollevi et al. Anesthesiology 1984, OKT:400. 